\title{
Ectopic eruption of the maxillary first permanent molar: a review and case report
}

\begin{abstract}
Ectopic eruption of the maxillary first permanent molar considered a common presentation during the mixed dentition stage. The prevalence of ectopic eruption has been reported in several studies. Multiple factors have been discussed in the literature that could result or contribute in the occurrence of this anomaly. Ectopic eruption may result in premature loss of primary second molar, loss of space for the erupting premolar leading to its impaction. Different management techniques have been discussed in the literature including early interceptive treatment. The purpose of this paper to briefly review the prevalence, etiologic factors and management of ectopic eruption. Moreover, the case report demonstrated that early diagnosis could minimize the need for active interceptive treatment even in moderate severity ectopic eruption cases that include the resorption of the distal root and pulpal involvement of the primary second molar
\end{abstract}

Keywords: Ectopic Eruption, First permanent molar, Primary dentition, Mixed dentition, Early diagnosis

\author{
Volume 9 Issue 2 - 2018
}

\begin{abstract}
Zain Z Hafiz
Department of Pediatric Dentistry and Orthodontics, King Saud University, Saudi Arabia
\end{abstract}

Correspondence: Zain Z Hafiz, Department of Pediatric Dentistry and Orthodontics, College of Dentistry, Riyadh, Saudi Arabia,Tel +966-I I-46948 I4, Email zhafizl@ksu.edu.sa

Received: March 16, 2018 | Published: April 02, 2018

\section{Abbreviation: EAL, Electronic Apex Locator \\ Introduction}

Tooth eruption is defined as the movement of the tooth from its intra-osseous site of development to its functional position in the oral cavity. ${ }^{1}$ Eruption is a complex process in which many factors contribute for a successful normal eruption. Furthermore, Although permanent teeth eruption is under significant genetic control, various general factors such as gender, socioeconomic status, craniofacial morphology, body composition can influence this process. ${ }^{2}$ Ectopic Eruption may be defined as the eruption of permanent tooth, which takes place in such a manner that partial or total resorption of the root(s) of an adjacent primary tooth occurs and can be noted during routine dental radiographic evaluation. ${ }^{3}$ Nikiforuk defined ectopic eruption as a process in which the permanent successor, due to deficient jaw growth or part of the jaw, takes a path of eruption that affects the predecessor tooth, leading to its premature loss resulting in malposed permanent tooth. ${ }^{4}$ Ectopic eruption of the first permanent molars is a local disturbance characterized by their eruption under the distal part of the second primary molars and failure of the first permanent molars to erupt normally. ${ }^{5}$ It occurs 25 times more often in the maxilla than mandible. ${ }^{6}$ If the ectopic eruption received no treatment it may cause:

a. Space loss for the erupting permanent premolar

b. Malocclusion

c. Decreased arch length. ${ }^{7}$

The premature loss of maxillary second primary molar may allow serious forward movement and tilting of the adjacent first permanent molar. Therefore when a primary second molar prematurely lost, fitting a space maintainer should be taken into consideration. ${ }^{8}$ Early diagnosis and the early start of treatment using the natural forces of eruption allow the prevention of more complicated malocclusions. ${ }^{9}$ The aim of this study is to briefly review the prevalence, etiology and management techniques used for treating ectopically erupting first permanent molar. Moreover, a radiographic case report is presented for an ectopically erupting maxillary right first permanent molar in a 7.5-year-old female patient.

\section{Literature review}

\section{Prevalence}

Chapman (1923) was one of the earliest to report about the ectopic eruption. He mentioned four cases of ectopic eruption of the maxillary first permanent molars in which he described four possible factors:

a. small arches

b. deviant paths of eruption of the permanent molar

c. lack of forward movement of all primary teeth

d. early eruption of the maxillary first permanent molars. ${ }^{10}$

Young observed in the 1619 children involved in his study that $3 \%$ were affected by the ectopic eruption of the maxillary first permanent molar. There was non-significant difference in the occurrence in the right or left side. He also reported that two-thirds of the ectopically erupting first permanent molars did not need any interceptive orthodontic treatment and erupted in their normal position in the oral cavity. ${ }^{6}$ A study by Carr \& Mink (1965) reported that $25 \%$ higher prevalence in cleft lip and palate patients. ${ }^{11}$ Bjerklin \& Kurol (1981) studied the prevalence of ectopic eruption of the maxillary first permanent molar in 2,903 children and they found that it affects $4.3 \%$ for the population and $21.8 \%$ for cleft children. ${ }^{12}$ Moyers (1992) reported that 3\% of American children present with this eruption disturbance. Moreover, siblings of affected children experience this eruption disturbance five times more than the other 
population..$^{13}$ Bierkline (1994) reported that siblings of those children who also had ectopic eruption showed a prevalence of $19.8 \%$, indicating a genetic background. ${ }^{14}$ This eruption disturbance showed gender predominance where it was observed more frequently in boys than in girls. ${ }^{11-15}$ However, Chintakanon (1998) studied the ectopic eruption of maxillary and mandibular first permanent molars in 3612 children. It has been found that 27 children had ectopic eruption of the maxillary first permanent molar for a prevalence rate of $0.75 \%$, which was higher in males than females, but the difference was not statistically significant $(p>0.05)$. It was also reported that there was no statistical difference between the left, right, or both sides of the arches. ${ }^{16}$ Furthermore, Barberia-Leache et al. (2005) reported that $4.3 \%$ of the studied subjects had ectopic eruption of the maxillary first permanent molar with equal occurrence in in both genders. Out of these ectopic eruptions $36.4 \%$ were unilateral and $63.6 \%$ bilateral with a right:left relation of $3: 1 .{ }^{17}$ Afify and Zwawi (2012) reported the prevalence of dental anomalies in the western region of Saudi Arabia. They've collected 878 digital orthopantomograms (OPGs) from their patients. They mentioned that two patients $(0.2 \%)$ had ectopic eruption of the third molar and 1 patient $(0.1 \%)$ had this disturbance in the lower premolar. ${ }^{18} \mathrm{Up}$ to the author's knowledge there are no studies on the prevalence of ectopic eruption of the maxillary first permanent molar in Saudi children.

\section{Etiological factors}

Clinically, it is crucial to determine whether any etiologic factors alone or combined cause the ectopic eruption of the maxillary first permanent molar. ${ }^{19}$ The etiology of ectopic eruption is a disturbance in the pattern of the individual's differential growth. Tissues and organs grow at different rates and times. Normal balance exists between the timing and rate of growth. Differential growth is the basis for normal progression of various physiologic processes as the eruption of teeth. Thereby, if this balance is affected by congenital or environmental interferences, the abnormality appears. Furthermore, Yaseen et al. (2011) mentioned that

1. a disturbance in the balance between the rate of jaw growth

2. the rate of eruption of the first molars

3. sizes of the teeth produces ectopic eruption may lead to the ectopic eruption of the maxillary first permanent molar. ${ }^{7}$

Different etiologic factors may lead to ectopic eruption. ${ }^{3-20}$ Genetic factor has been reported. ${ }^{5}$ Bjerklin and Kurol (1983) discussed the attribution of local factors that may cause this anomaly including:

1. larger permanent molars

2. pronounced mesial angle of eruption

3. crowding

4. a tendency for shorter maxilla

5. abnormal shape of the second primary molar crown. ${ }^{21}$

Chintakanon (1998) reported that caries status of maxillary second primary molar is a minor factor that may cause the ectopic eruption of maxillary first permanent molars. Moreover, the study showed that most common etiologic factors was the path of eruption and mesial angulation of the first permanent molar relative to the chosen reference lines, while the size of the second primary molars was an etiologic factor significant only for the ectopic eruption of mandibular first permanent molars. ${ }^{5}$ Likewise, factors that may lead to this anomaly are discrepancies in bone-tooth size or a disturbance in the chronology of bone growth at the tuberosity region in relation to the calcification and eruption of the molar. Additionally, dental factor like unfavorable morphology of second primary molar crown may attribute in this eruption anomaly. Heredity factor should also be considered. ${ }^{17}$

\section{Management}

Ectopic eruption according to Young's (1957) classification is two types:

A. reversible (jump)

B. irreversible (hold). ${ }^{6}$

The prevalence of the reversible pattern occurs in almost $66 \%$ of ectopically erupting permanent maxillary first molars. Moreover, the ectopically erupting permanent first molar moves spontaneously from a locked position and erupts into normal plane. In the irreversible form, the permanent first molar is in a locked position until interceptive treatment is performed or the primary second molar is being prematurely lost. Furthermore, Ectopic eruption is classified based in its effect on the primary second molar tooth;

1. Grade 1: mild causing limited resorption to the cementum or with minimum dentine penetration of the primary second molar

2. Grade 2: moderate causing resorption of the dentine without pulp exposure of the primary second molar

3. Grade 3: severe causing resorption of the distal root leading to pulp exposure of the primary second molar

4. Grade 4: very severe causing resorption that affects the mesial root of the primary second molar. ${ }^{17}$

Many treatment modalities for treating ectopic eruption have been discussed. Fifty to sixty nine percent of the cases of ectopically erupting permanent molars correct spontaneously without intervention. ${ }^{6}$ Several management techniques that were suggested in the literature ranged from orthodontic band and springs, ${ }^{22}$ Croll's bilateral band, wire appliance and Grim's removable Hawley appliance with a kick spring to distalize the locked molar. The purposes of treatment of ectopic first molar are movement of the tooth distally to its normal position in occlusion and correction of the mesial angulation to regain space for normal eruption of the second premolar. ${ }^{23}$ Majority of cases with spontaneous self-correction occurs before the age of seven. ${ }^{17}$ Hennessy et al. (2012) reported that the treatment of ectopically erupting first permanent molar depends on several factors including: (a) patient's age: in patients under the age of eight it is recommended to do a six-month follow up. If the case doesn't get self-corrected then interceptive treatment is required to avoid the root resorption of the primary second molar, (b) status of the second primary molar: when the primary molar shows symptoms of irreversible pulpitis or increased mobility, extraction must be considered. Expected space loss after extraction can be prevented using a suitable space maintainer, (c) Presence of the second premolar: In cases where the second premolar is congenitally missing during OPG interpretation, it's advisable after consulting with a specialist to extract the primary second molar and allow the first permanent molar to drift mesially and close the space, (d) and Severity of impaction: as mentioned before, ectopic eruption is 
classified into 4 grades from 1-4, depending on their severity. Grade 1 ectopic molars should be followed up and give them a chance to selfcorrect spontaneously. Grade 2 molars require interceptive treatment, which include interproximal wedging or distal tipping. Grade 3 molars may require the removal of the second primary molar and are treated through active distal tipping of the ectopic permanent molar by using a removable appliance or by placing fixed brackets. Finally, grade 4 ectopic eruption is so severe in which it involves resorption of the mesial root of the second primary molar. Hereby; extraction of the primary molar is recommended after the occlusion is being assessed by an expert to prevent loss of space. ${ }^{23}$ In General, treatment methods can be classified into two categories:

1. Interproximal wedging,

2. Distal tipping.

\section{Interproximal wedging}

It aims to place a separating medium between the second primary molar and first permanent molar, used in cases of reversible type of ectopic eruption on the distal surface of the primary second molar, ${ }^{24}$ such as: metal or elastic seperators, ${ }^{25}$ kesling separator, deimpactors, ${ }^{26}$ helical springs ${ }^{27}$ and brass ligature wire. ${ }^{28}$ Kupietzky (2000) treated a six-year-old patient who had bilateral ectopic eruption of the maxillary first permanent molars, which were corrected in 8-month period after using the brass wire which was first presented by Levitas in $1964 .{ }^{29} \mathrm{As}$ well, Yong and Ki Tae et al. (2005) managed a case of ectopic eruption using triangular wedging spring. The case was successfully treated in 2 months and suggested that using triangular wedging spring can be a simple, less irritable, and more effective way of correcting ectopic eruption of the permanent first molar. ${ }^{28}$ In addition, Hennessy et al. (2012) reported a successful treatment of an ectopically erupting maxillary first permanent molar using elastomeric separator. ${ }^{23}$

\section{Distal tipping}

Distal movement of the first permanent molar is required in cases of severe resorption of the primary molar, and the permanent molar has drifted mesially. ${ }^{23}$ Kurol and Bjerklin (1984) treated 46 patients with ectopic eruption using Kloehn type of cervical headgear. The mean age at the start of treatment was 8.3 years and the mean treatment time was 0.8 year. The treatment resulted in distal tipping of the first permanent molar to a good occlusion in all children. They found that the most favorable time for treatment seems to be when the second premolar is close to eruption or erupting at the end of the treatment period. ${ }^{30}$ Moreover, Weinberger (1992) used a fixed appliance to treat bilateral ectopic eruption of the maxillary first permanent molars. In two months the first permanent molars were in their normal places and the fixed appliance was left for additional one month to avoid the relapse that could occur. ${ }^{31}$ Kinzinger et al. (2006) used the pendulum appliance that allows for rapid molar distalization without the need for patient compliance. Twenty-nine patients in the mixed dentition have received a modified pendulum appliance with a distal screw and a preactivated pendulum spring for bilateral distalization of the maxillary molars. The patients were divided into 4 groups based on dentition stages: patient group 1 was in the early mixed dentition; patients had resorption of the distal root areas of the deciduous molars being used for dental anchorage, and the unerupted premolars were located at the distal margin of the deciduous molar root region. Based on radiographs taken before placement of the pendulum appliance, patient group 2 was diagnosed as having a central location of the unerupted premolars. In the third group, the first premolars were already erupted and could be integrated into the dental anchorage, but the canines were not yet erupted. In the fourth group, the first premolars and both canines were fully erupted. They found that in patients being treated with pendulum appliances, the anchorage quality of the deciduous molars that were already partially resorbed in the distal root area was reduced and the mesial drift of the deciduous molars and incisors was increased, without impairing the extent and quality of the molar distalization. ${ }^{32}$

\section{Case report}

Seven and a half year old female patient, presented to the pediatric dental clinic complaining of irregular, mild pain and pressure on the upper right area of her teeth. Medical history was irrelevant. Extraoral and intra-oral examinations including periodontal examination were within normal limits. Oral examination shows that the patient is in the mixed dentition stage and all first permanent molars are fully erupted except for the maxillary first right permanent molar. $\mathrm{X}$-rays were requested including (OPG, periapical and bitewing) for the area of tooth \#16. Although it was explained to the parent that using a thyroid collar during OPG exposure would affect the quality of the X-ray, the parent requested to cover her child's neck with the thyroid collar. Since it was found that the collar would not affect the interpretation of the area of interest, the request was approved. Radiographic interpretation revealed an ectopic eruption of the maxillary right first permanent molar accompanied with resorption of maxillary second molar (Figure 1). Moreover, examination of the primary molar showed no mobility, mild sensitivity to percussion and palpation. However, the resorption almost compromised the pulp integrity of the primary second molar. The distal root of the primary molar was totally resorbed and the crown of the first permanent molar was locked under the distal part of the crown of primary molar. The treatment's goal in this case was to correct the eruption path of the first permanent molar without using active interceptive orthodontic techniques. Treatment plan was discussed with an orthodontist and approved that the removal of the primary molar is required and reverse band and loop should be placed to maintain the space for the maxillary second premolar and guide the eruption of the first permanent molar.
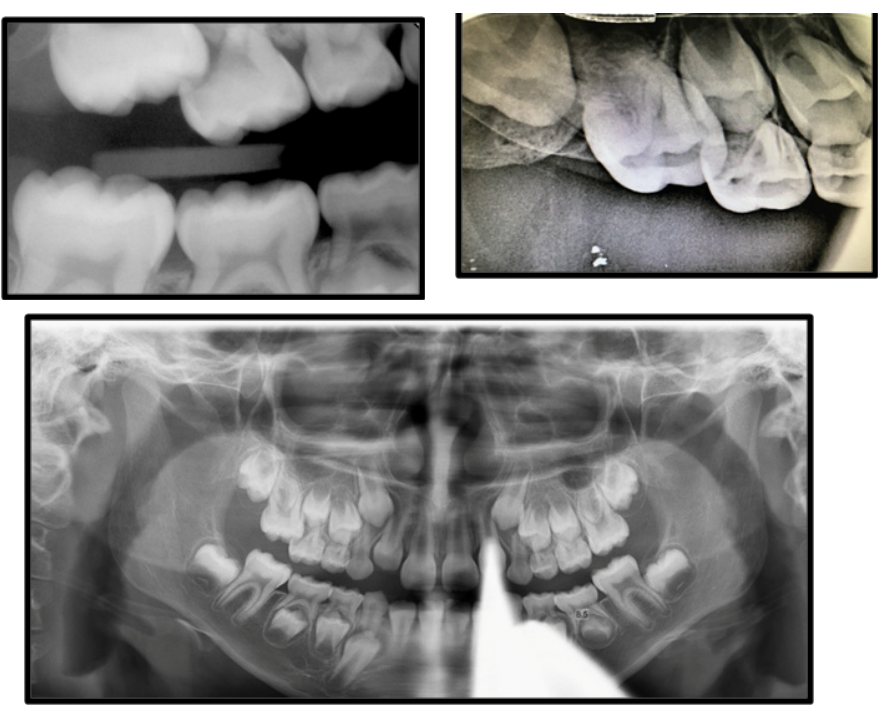

Figure I Preoperative X-rays. 


\section{Treatment}

The treatment plan was discussed and clearly explained to the parent who signed the consent form. The patient received topical anesthesia (Gingi-Pak, CA, USA) followed by local anesthesia $2 \%$ xylocane 1:100.000 epinephrine (Dentsply Ltd, Surrey, England) infiltration at the maxillary primary right upper second molar area. The primary molar was extracted (Figure 2) and post extraction instructions were given to the parent (Figure 3). After one week, reverse band and loop was constructed and placed where the band was placed on the maxillary right first primary molar and the loop was touching the mesial aspect of the crown of maxillary first permanent molar. The case was followed and after 8 months the primary first molar exfoliated and the permanent first molar was fully erupted in correct occlusion with available space for the eruption of the premolars (Figure 4). After 1.5 years follow up, the first and second premolars were fully erupted and the patient has normal occlusion with no further treatment needed (Figure 5).

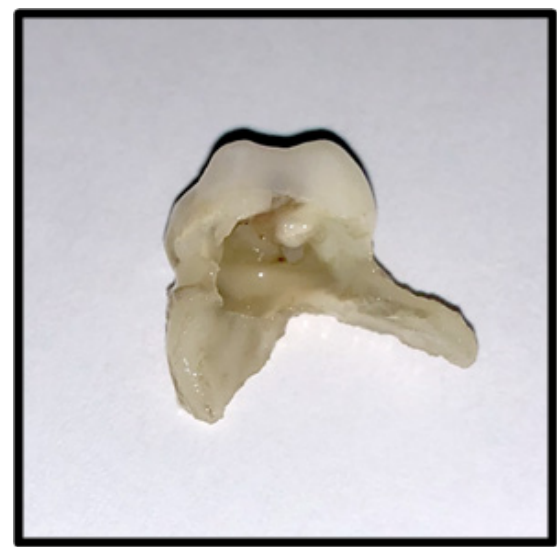

Figure 2 Maxillary primary second molar after extraction showing complete resorption of the distal root with pulpal involvement.
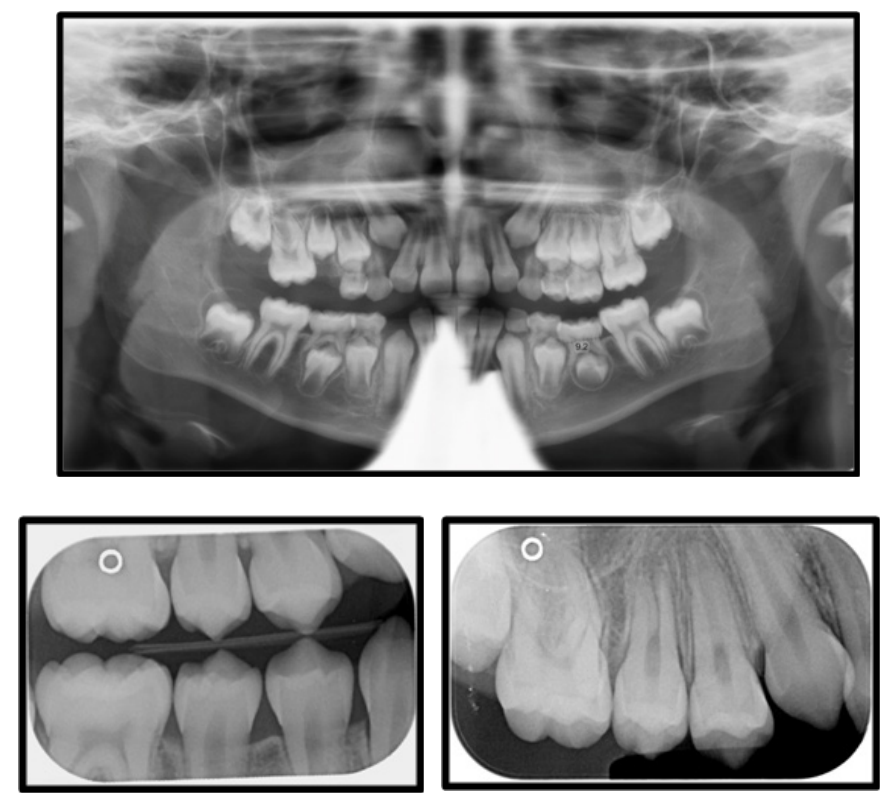

Figure 3 Post extractions X-ray.

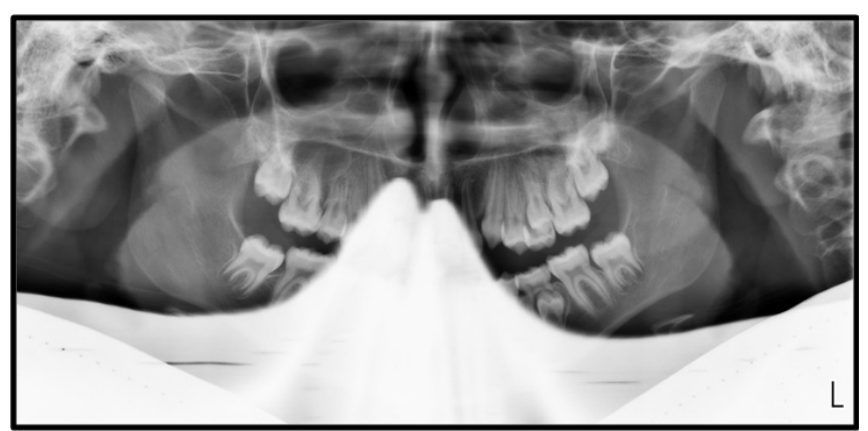

Figure 4 One year and a half post extraction X-rays.

\section{Conclusion}

Early intervention of ectopically erupting first permanent molars is very crucial to avoid complex orthodontic treatment later on. Although in this case the amount of resorption in the primary second molar was grad III, the ectopic eruption was successfully treated by extracting the primary second molar and guiding the eruption of the first permanent molar using space maintainer which is considered simple and more convenient to use with children.

\section{Acknowledgment}

None.

\section{Conflict of interest}

None.

\section{References}

1. Massler M, Schour I. Studies in tooth development: theories of eruption. Am J Orthodont Oral Surg. 1941;27(10):552-576.

2. Almonaitiene R, Balciuniene I, Tutkuviene J. Factors influencing permanent teeth eruption. Part one - general factors. Stomat Baltic Dent Maxillofac J. 2010;12(3):67-72.

3. O'Meara W. Ectopic eruption pattern in selected permanent teeth. J Dent Res. 1962;41(3):607-616.

4. Nikiforuk G. Ectopic Eruption: Discussion and clinical report. J Ont Dent Assoc. 1948;25:243-246.

5. Chintakanon K. Ectopic eruption of the first permanent molars: prevalence and etiologic factors. Angle Orthod. 1998;68(2):153-159.

6. Young D. Ectopic eruption of permanent first molar. J Dent Child. 1957;24:153-162.

7. Yaseen S, Naik S, Uloopi K. Ectopic eruption - A review and case report. Contemp Clin Dent. 2011;2(1):3-7.

8. Avramaki E, Stephens C. The effect of balanced and unbalanced extraction of primary molars on the relationship of incisor centre lines- A pilot study. J Ped Dent. 1988;4(1):9-12.

9. Braham R, Morris M (1980) Textbook of Pediatric Dentistry. Md: Williams and Wilkins, 1st ed., Baltimore, 329-331.

10. Chapman H. First upper permanent molar partially impacted against second deciduous molar. Int J ortho oral surg and Radio. 1923;9(5):339-345.

11. Carr E, Mink J. Ectopic eruption of the first permanent maxillary molar in cleft lip and cleft palate children. ASDC J of Dent Child. 1965;32(1):179-188. 
12. Bjerklin K, Kurol J. Prevalence of ectopic eruption of the maxillary first permanent molar. Swed Dent J. 1981;5(1):29-34.

13. Moyers R. (1992) Manual de Ortodoncia. $4^{\text {th }}$ ed. Editorial Medica Panamericana, Buenos Aires; 129.

14. Bjerklin K. Ectopic eruption of the maxillary first permanent molar. An epidemiological, familial, etiological and longitudinal clinical study. Swed Dent J. 1994;100:1-66.

15. Kimmel N, Gellin M, Bohannan H, et al. Ectopic eruption of maxillary first permanent molars in different areas of the United States. ASDC J Dent Child. 1982;49(4):294-299.

16. Chintakanon K. Ectopic eruption of the first permanent molars: prevalence and etiologic factors. Angle Orthod. 1998;68(2):153-160.

17. Barberia-Leache E, Suarez-Clúa M, Saavedra-Ontiveros D. Ectopic eruption of the maxillary first permanent molar: characteristics and occurrence in growing children. Angle Orthod. 2005;75(4):610-615.

18. Afify A, Zawawi K. The prevalence of dental anomalies in the western region of Saudi Arabia. ISRN Dent. 2012;1-5.

19. Kurol J, Bjerklin K. Resorption of maxillary second primary molars caused by ectopic eruption of the maxillary first permanent molar: a longitudinal and histological study. ASDC J Dent Child. 1982;49(4):273-279.

20. Halterman C.A Simple Technique for the Treatment of Ectopically Erupting Permanent First Molars. J Am Dent Assoc. 1982; 105(6):1031-1033.

21. Bjerklin K, Kurol J. Ectopic eruption of the maxillary first permanent molar: Etiologic factors. Am J Orthod. 1983;84(2):147-155.

22. Bjerklin K, Kurol J. Treatment of children with ectopic eruption of maxillary first permanent molar by cervical traction. Am J Orthod. 1984;86(6):483-492.
23. Hennessy J, Al-Awadhi E, Dwyer L, et al. Treatment of ectopic first permanent molar teeth. Dent Update. 2012;39(9):656-658.

24. Weinberger S. Correction of bilateral ectopic eruption of first permanent molars using a fixed appliance. Pediatr Dent. 1992;14(6):382-383.

25. McDonald R. Dentistry for the Child and Adolescent. $5^{\text {th }}$ ed. St. Louis, MO: Mosby; 1985;771-780.

26. Sharma P, Rypel T. Ectopic eruption of permanent molars and their management. Quint Int. 1978;9(12):47-52.

27. Levitas T. A simple technique for correcting an ectopically erupting maxillary first permanent molar. J Dent Child. 1964;31:16-18.

28. Yong Heon K, Ki Tae P. Simple Treatment of Ectopic Eruption With a Triangular Wedging Spring. Ped Dent. 2005;27(2):143-145.

29. Kupietzky A. Correction of ectopic eruption of permanent molars utilizing the brass wire technique. Pediatr Dent. 2000;22(5): 408-412.

30. Kurol J, Bjerklin K. Treatment of children with ectopic eruption of the maxillary first permanent molar by cervical traction. Am J Orthod. $1984 ; 86(6): 483-492$.

31. Weinberger S. Correction of bilateral ectopic eruption of first permanent molar using a fixed appliance. Ped Dent. 1992;14(6):382-383.

32. Kinzinger G, Wehrbein H, Gross U, et al. Molar distalization with pendulum appliances in the mixed dentition: Effects on the position of unerupted canines and premolars. Am J Orthod Dentofacial Orthop. 2006;129(3):407-417. 